\title{
UN PROBLEMA EN LAS TESIS FUNDAMENTALES DEL DERECHO QUE SUPONE EL POSITIVISMO JURÍDICO ESTILO HART
}

\section{BERNARDo ANDRÉs GALlEgos MENDOZA ${ }^{1}$}

RESUMEN: En este artículo defenderé la tesis de que el positivismo jurídico estilo Hart aún no cuenta con una formulación adecuada de las tesis fundamentales del derecho. Consideraré, al menos, tres formulaciones de tesis fundamentales del derecho proporcionadas por algunos positivistas jurídicos y concluiré que ninguna es correcta, ya que ellas están enfocadas en las proposiciones sobre el derecho sin ofrecer explicación alguna de cuáles son los casos que podrían estar indeterminados. Este problema es patente cuando distinguimos entre proposiciones sobre el derecho y, lo que llamaré, proposiciones jurídicas ordinarias. Analizaré dos posibles enmiendas a las tesis fundamentales del derecho que un positivista jurídico podría ofrecer, pero ninguna será satisfactoria. Finalmente, apuntaré que una tesis muy plausible de la lógica de la verdad muestra que no sólo las tesis fundamentales del derecho, sino incluso los fundamentos del derecho del positivismo jurídico estilo Hart requieren una corrección.

PALABRAS CLAVE: fundamentos del derecho, positivismo jurídico, validez jurídica, indeterminación jurídica, lógica de la verdad.

ABSTRACT: In this paper I will defend the thesis that Hart's style legal positivism does not yet have an adequate formulation of the fundamental thesis of law. I am going to consider, at least, three formulations of fundamental thesis of law provided for some legal positivists and I will conclude that none is correct because they are all focused in the propositions about law without offering any account of the cases could be indeterminate. This problem is

1 Realizó estudios de derecho y filosofía en la UNAM. Ha impartido las cátedras de Teoría Jurídica Contemporánea I y Lógica I en el Posgrado de Derecho de la UNAM. Actualmente realiza su investigación doctoral sobre la objetividad interna en el derecho adscrito a la Facultad de Filosofía y Letras de la UNAM. Correo electrónico: <bernardogallegosm@gmail.com>. 
showed when we distinguish between propositions about law and, what I will call, ordinary propositions of law. I will analyse two possible amendments of the fundamental thesis of law that a legal positivist could offer, but none of them will be satisfactory. Finally, I will point out that a very plausible thesis of the logic of truth shows that not only the fundamental thesis of law, but even the grounds of law of Hart's style legal positivism require an amendment.

KeYWoRDS: grounds of law, legal positivism, legal validity, legal indeterminac, logic of truth.

Sumario: I. Tesis fundamentales y fundamentos del derecho. II. La tesis fundamental del derecho asumida por el positivismo jurídico estilo Hart. III. Una insuficiencia del positivismo jurídico estilo Hart. IV. El fracaso de dos estrategias de solución. V. Proposiciones sobre el derecho y lógica de la verdad. VI. Conclusiones. VII. Fuentes.

\section{i. Tesis fundamentales y fundamentos del DeRecho}

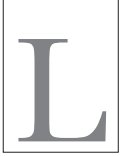

a pregunta de qué es el derecho puede ser respondida, en gran medida, explicando cómo se determina el derecho. Encontrar una respuesta a esta última pregunta puede conducirnos a cuestionarnos cómo una norma adquiere validez jurídica² o, de manera equiparable, cómo es que una proposición jurídica es verdadera. ${ }^{3}$ Por el momento, llamaré "tesis fundamentales del dere-

2 Además de Jules Coleman, que en breve veremos, también puede consultarse a John Gardner, Scott Shapiro y Andrei Marmor ( $c f r$. John Gardner, Law as a Leap of Faith: Essays on Law in General, Oxford, Oxford University Press, 2012, pp. 19-20; Scott Shapiro, Legality, Cambridge, Massachusetts, Harvard University Press, 2011, pp. 8-12 (33-38); y Andrei Marmor, Philosophy of Law, Princeton, Princeton University Press, 2011, p. 126). De aquí en adelante, cuando se cite una obra extranjera y exista una traducción al español publicada, se colocará(n) entre paréntesis la(s) página(s) correspondientes de la versión en español (salvo que la traducción publicada admita serias controversias).

3 Así interpretan la cuestión principalmente Ronald Dworkin y Joseph Raz (cfr. Ronald Dworkin, Law's Empire, Cambridge, Massachusetts, Harvard University Press, 1986, cap. 1 y Joseph Raz, The Authority of Law: Essays on Law and Morality, 
cho" a las propuestas explicativas que se han ofrecido o se pueden ofrecer al respecto. ${ }^{4}$ El positivista jurídico, John Austin, por ejemplo, afirmó que el derecho estaba constituido por mandatos generales emitidos por el soberano (alguien que habitualmente es obedecido y no obedece a nadie más). Si interpretamos que lo que Austin afirmaba era que las leyes adquirían su validez jurídica en virtud de ser mandadas por el soberano, entonces estamos frente a una tesis fundamental del derecho. Otro ejemplo de tesis fundamental del derecho es la propuesta por el antipositivista jurídico, Ronald Dworkin. Mantuvo que las proposiciones de derecho "son verdaderas si figuran o surgen como consecuencia de principios de justicia, equidad y debido, que proceso que proporcionan la mejor interpretación constructiva de la práctica legal de la comunidad", 5 ofreciéndonos, con ello, una explicación de qué hace que las proposiciones jurídicas sean verdaderas, una cuestión equiparable a la de la validez jurídica de las normas.

Estos ejemplos nos sirven para ilustrar propuestas de tesis fundamentales del derecho y, a su vez, también para considerar posibles fundamentos del derecho. ${ }^{6}$ Austin, por ejemplo, asume que los fundamentos del derecho son los mandatos del soberano y Dworkin, en cambio, afirma que dichos fundamentos no son esos hechos sociales, sino la mejor interpretación moral del derecho. ${ }^{7}$ El presente

Oxford, Oxford University Press, 1979, cap. 4). También se ha entendido de este modo a Hart y en general al positivismo jurídico ( $c f r$. Dennis Patterson, Law and Truth, Oxford, Oxford University Press, 1996, pp. 59 y 64).

4 Más adelante el concepto de tesis fundamental del derecho adquirirá mayor extensión.

5 Ronald Dworkin, op. cit., p. 225 (164).

6 Por el momento, entenderé que los fundamentos del derecho son aquellas condiciones en virtud de las cuales una norma adquiere validez jurídica, o bien, una proposición jurídica es verdadera. Más adelante el concepto de fundamento del derecho adquirirá mayor extensión.

7 Según la exposición se debe distinguir entre tesis fundamentales del derecho y fundamentos del derecho. Dworkin mismo, así como importantes positivistas 
artículo, sin embargo, no destaca ninguna importancia particular de alguna de estas dos propuestas, sino sólo la de funcionar como ejemplos introductorios de tesis fundamentales y fundamentos del derecho. El objetivo central de la exposición será, en cambio, discutir las tesis fundamentales del derecho que algunos positivistas jurídicos relevantes suponen. El desarrollo será como sigue: primero expondré algunas de las líneas centrales del positivismo jurídico estilo Hart y tres versiones de la tesis fundamental del derecho que plausiblemente podría asumir. En segundo lugar, argumentaré que dichas versiones son insuficientes para explicar el derecho. En tercer lugar, analizaré dos posibles enmiendas para un adecuado planteamiento, llegando, sin embargo, a la conclusión de que ninguna es satisfactoria. Finalmente, exploraré una consecuencia que un axioma sobre la verdad podría implicar para el positivismo jurídico estilo Hart, observando que los fundamentos del derecho que propone requieren alguna corrección.

\section{LA TEsis fundamental del deREcho ASUMida por EL POSITIVISMO JuRÍdico ESTILO HART}

Hart propuso una teoría del derecho basada en la observación de que existen dos puntos de vista que podemos tener frente a la regularidad de una conducta: el interno y el externo. El primero es aquel que tiene algún miembro de la comunidad al aceptar reglas. ${ }^{8} \mathrm{Y}$, el segundo aquel que tiene un espectador quien, aunque no

que se refieren explícitamente al tema, tales como Wilfrid Waluchow y Scott Shapiro, utilizan solamente la frase "fundamentos del derecho" (grounds of law) tanto para referirse a las tesis fundamentales del derecho como a los fundamentos mismos y dejan que el contexto aclare su significado (cfr. Ronald Dworkin, op. cit., pp. 3-6 (16-19); Wilfrid Waluchow, Inclusive Legal Positivism, Oxford, Oxford University Press, 1994, pp. 9-11 (23-26) y Scott Shapiro, op. cit., pp. 284-287 (347-350).

${ }^{8}$ Casos claros que evidencian la aceptación de una regla como estándar de conducta son aquellos donde se usa para valorar la validez de otra regla o para valorar que alguien tiene una obligación o derecho subjetivo. 
acepte una regla como estándar de conducta, puede, por ejemplo, observar que otros lo hacen o simplemente observar que existe una regularidad de conducta. Según Hart, ignorar el punto de vista interno es lo que mantenía oscurecido el concepto de derecho hasta entonces. Lo importante de este punto de vista, entre otras cosas, es que nos capacita para explicar la existencia de reglas jurídicas últimas en una sociedad; en especial de algo que Hart llamó la "regla de reconocimiento", una regla secundaria de carácter social. Su carácter secundario se debe a que no trata de conductas sino de reglas, ya que tiene por objeto señalar los criterios del derecho válido; es decir, una sección paradigmática del derecho conformada, en especial, por reglas jurídicas primarias que señalan cómo se deben comportar aquellas personas sometidas a su jurisdicción. Y su carácter social se debe a que su existencia ocurre en virtud de una práctica: la regularidad de una conducta que aceptan, como pauta de comportamiento, los agentes pertinentes de una comunidad. ${ }^{9}$

Una observación importante que añade Hart a la existencia de los puntos de vista interno y externo es que entre nuestras expresiones han de distinguirse los enunciados jurídicos internos de los externos. El tipo de casos al que más recurre para ilustrar los primeros son los enunciados de validez jurídica que resultan de usar la regla de reconocimiento (o una regla identificada por ella). Un ejemplo sería: "La prohibición de conducir un vehículo a más de 80 $\mathrm{km}$ por hora es derecho válido". ${ }^{10}$ Por otra parte, como enunciados jurídicos externos podemos considerar a aquellos que predicen

9 En los sistemas jurídicos nacionales modernos, que para Hart eran los casos paradigmáticos de derecho, los agentes pertinentes son los funcionarios (officials).

10 Hart sugiere que existen al menos tres tipos de enunciados jurídicos internos adicionales: i) aquel cuyos enunciados atribuyen una obligación jurídica a alguien (cfr. H. L. A. Hart, The Concept of Law, 2a. edición, Oxford, Oxford University Press, 1994, pp. 56, 57, 89, 90 y 218 (71, 72, 112 y 269 de El Concepto de Derecho); ii) aunque no tan explícito en ello, otro sería aquel cuyos enunciados atribuyen un acto jurídico a alguien; y, iii) aquel cuyos enunciados adquieren autoridad definitiva (para estos dos últimos tipos, véase la discusión que Hart emprende contra 
que frente a ciertas conductas ocurrirán ciertas reacciones hostiles o bien aquellos que describen que ciertos agentes mantienen el punto de vista interno respecto de alguna regla. Ejemplos de tales enunciados externos podrían ser, respectivamente, los siguientes: i) cuando se enciende la luz roja de un semáforo, los conductores detendrán sus vehículos; y ii) en México los funcionarios aceptan las normas creadas por los constituyentes originales que no han sido aún reformadas.

Nótese que esta clasificación no implica que toda regla apoyada por el punto de vista interno puede ser declarada a través de un enunciado jurídico interno; la regla de reconocimiento es un ejemplo de ello. Ya que su existencia sólo se puede explicar por la regularidad de una conducta y el punto de vista interno, se sigue que no existe una regla ulterior que pueda usarse para identificar la regla de reconocimiento como regla válida. Por esta razón, Hart enfatiza que la regla de reconocimiento no es válida ni inválida y que su existencia sólo puede expresarse a través de un enunciado jurídico externo. ${ }^{11}$

un argumento del escepticismo ante las reglas, especialmente en Hart, op. cit., pp. 141-144 (176-179 de El Concepto de Derecho).

11 Cfr. Ibidem, pp. 110, 292 y 293 (137, 310 y 311 de El Concepto de Derecho). Raz mantiene la lectura de que los enunciados jurídicos internos de Hart expresan la aceptación de la regla sobre la que tratan (cfr. Joseph Raz, "H. L. A. Hart (1907-1992)", en Utilitas, vol. 5, n² 2, 1993, p. 148). Más recientemente, Kevin Toh interpreta que para Hart los enunciados jurídicos internos presuponen la aceptación de la regla sobre la que tratan. Toh se enfrenta con los pasajes de las páginas 110, 292 y 293 de The Concept of Law y, en su opinión, los encuentra incongruentes con otras partes de la obra de Hart, llegando a la conclusión de que es mejor ignorar esas afirmaciones ( $c f r$. Kevin Toh, "Hart's Expressivism and his Benthamite Project", en Legal Theory, $n^{\circ}$ 11, 2005, pp. 90-91; y, Kevin Toh, "An Argument against the Social Fact Thesis", en Law and Philosophy, no 27, 2008, parte XI). Me parece que Raz y Toh se equivocan, dado que la introducción de enunciados jurídicos internos no tiene, en la teoría de Hart, la función de explicar la expresión de aceptación de las reglas de la que ellos tratan, sino explicar cómo es que a través de ellos se muestra o expresa la aceptación de una regla distinta (en especial, la regla de reconocimiento) y cómo es que puede existir una enorme 
De acuerdo con esta explicación, no sólo el punto de vista interno que un grupo de participantes puede tener sobre una regla de reconocimiento (o sobre cualquier otra regla) elucida el concepto de derecho, sino que, concomitante a ello, también los enunciados jurídicos internos que podemos formular en virtud de una regla de reconocimiento lo hacen. Este último tipo de enunciados logra su contribución en la elucidación del concepto de derecho dado que define la amplia sección del derecho conformada por las reglas de las que se predica validez jurídica, ${ }^{12}$ y que está presente en los sistemas jurídicos nacionales modernos. Así, se puede entender que Hart mantuvo, como tesis fundamental del derecho, una que se puede expresar aproximadamente del modo siguiente:

\section{T1 de Hart}

Una regla es jurídicamente válida en una determinada comunidad, M, si y sólo si satisface ciertos criterios de validez jurídica. $^{13}$

cantidad de reglas jurídicas antes de que puedan ser practicadas (cfr. Hart, op. cit., p. 256 (33 y 34 del Post Scríptum al Concepto de Derecho)).

12 Los enunciados jurídicos internos hacen una contribución aún más amplia con el concepto de derecho, pues también definen los enunciados jurídicos internos de obligación y derecho subjetivo. Incluso, aunque menos explícitamente, todo indica que Hart supone que también definen los enunciados jurídicos internos que hacemos al mantener la ocurrencia de un acto jurídico. En la exposición central ignoraré estos dos últimos tipos de enunciados jurídicos internos. Por otro lado, tal vez la única parte en la que no contribuyen estos enunciados jurídicos internos en la elucidación del concepto de derecho es en la definición de la sección del derecho (si es que lo es) conformada por reglas sociales, como la regla de reconocimiento.

13 Hart mismo pareció siempre estar conforme con una teoría de la regla de reconocimiento que estableciera tanto condiciones suficientes como necesarias para la validez jurídica de las normas (cfr. Hart, op. cit., pp. 95, 103 (118, 128 y 129 de El Concepto de Derecho)). Shapiro también interpreta de este modo a Hart al mantener por un lado que "Cualquier norma que lleve una de las marcas de la autoridad establecidas en la regla de reconocimiento es una norma [law] de ese 
Jules Coleman, un positivista jurídico incluyente, ha afirmado una tesis fundamental del derecho con la que plausiblemente Hart estaría de acuerdo. Coleman expresó su tesis del modo siguiente: "una regla es una regla jurídica si posee la característica $C$; y un principio moral es un principio jurídico si posee la característica $C_{1}$. La regla de reconocimiento entonces establece que una norma es jurídica si y sólo si posee $C_{0} C_{1}{ }{ }^{14} \mathrm{Si}$ se quisiera equiparar esta tesis a la que he reconstruido de Hart, se podría reformular del modo siguiente:

\section{T1 de Coleman}

Una norma es jurídica (jurídicamente válida) en una determinada comunidad, $\mathrm{M}$, si y sólo si posee la característica $\mathrm{C}$ o la característica $\mathrm{C}_{1}$, según lo estipule su propia regla de reconocimiento.

sistema" (Shapiro, op. cit., p. 84 (120)); y, por otro, al decir que para Hart, una regla existe sólo en caso de que esté validada por la regla de reconocimiento ( $c f r$. Shapiro, op. cit., p. 90 (127)). Kenneth Himma, por otra parte, considera que tanto el positivismo jurídico incluyente como el excluyente se caracterizan por la tesis de que "por cada proposición $P, P$ es jurídicamente válida si y sólo si satisface los criterios articulados en la regla de reconocimiento convencional" (Kenneth Himma, "Inclusive Legal Positivism", en Jules Coleman y Scott Shapiro (eds.), Oxford Handbook of Jurisprudence and Philosophy of Law, Oxford, Oxford University Press, 2002, p. 143 (387)).

Por otra parte, una interpretación más amplia de T1 de Hart, podría incluir la consideración de enunciados jurídicos internos de obligación y derecho subjetivo, así como de actos jurídicamente válidos. Tal tesis podría ser la siguiente: una regla, obligación, derecho subjetivo o acto es jurídicamente válido en una determinada comunidad, M, si y sólo si satisface ciertos criterios de validez jurídica. Hago a un lado esta interpretación con el fin de facilitar la comprensión del problema central que pretendo mostrar.

14 Jules Coleman, "Negative and Positive Positivism", en The Fournal of Legal Studies, vol. 11, nº 1, 1982, p. 149 (la traducción es mía). 
Donde $\mathrm{C}_{1}$ es, para Coleman, algún criterio de validez jurídica no restringido a una fuente social. ${ }^{15}$

Joseph Raz, un positivista jurídico excluyente, consideró, en su famoso artículo "Legal Reasons, Sources and Gaps", ${ }^{16}$ la posibilidad de una tesis fundamental del derecho parecida a la de Hart aunque con ciertas especificaciones: i) en lugar de enfocarse sobre las condiciones de validez jurídica de las normas, lo hizo sobre las condiciones de verdad de las proposiciones jurídicas y ii) restringe los criterios de validez jurídica de una norma (ahora criterios de verdad de una proposición jurídica) a fuentes sociales. Veamos:

Ensayo de Raz:

"S(p) es una condición lógicamente necesaria y suficiente para la verdad de $\mathrm{p}$.

$\mathrm{S}(\neg \mathrm{p})$ es una condición lógicamente necesaria y suficiente para la falsedad de p". ${ }^{17}$

Donde S es la propiedad de tener una fuente (source), por ejemplo, haber sido legislado, ser la ratio decidendi de una decisión pasada, etc. $\mathrm{Y}$, por su parte, p es una variable que cubre proposiciones jurídicas; una de ellas podría ser que está jurídicamente prohibido conducir a más de $80 \mathrm{~km}$ por hora. ${ }^{18}$ Llamo a esta propuesta "Ensayo de Raz" porque no obstante su autor haya considerado estas tesis fun-

15 Coleman se explica del siguiente modo: "Supongamos que $C_{1}$ hace de la verdad moral una condición de legalidad, de modo que un principio moral no podría ser parte del derecho de una comunidad a no ser que fuera verdadero" (Coleman, op. cit., p. 152, la traducción es mía).

16 Raz, op. cit., The Authority ..., cap. 4.

17 Ibidem, p. 57 (82).

18 Más adelante se elaborará una distinción entre proposiciones sobre el derecho y, lo que llamaré, proposiciones jurídicas ordinarias; se comprenderá entonces que vale la pena interpretar que para Raz p es una variable que cubre proposiciones jurídicas ordinarias. 
damentales como las más apegadas a la tesis de las fuentes, ${ }^{19}$ acabó prefiriendo otra propuesta que más adelante será abordada.

En parte debido a que estas tres formulaciones comparten la suposición de que existen reglas de reconocimiento que dan identidad a las distintas jurisdicciones y en parte como una estipulación, consideraré que las tres mantienen el estilo Hart. El aspecto que quisiera destacar de estas tres formulaciones es la suposición compartida de que la validez jurídica de una norma (o, en su caso, la verdad de una proposición jurídica) está relacionada de modo bicondicional con sus respectivos criterios: en los tres casos se mantienen condiciones necesarias y suficientes para la validez jurídica de una norma o la verdad de una proposición jurídica.

El positivismo jurídico estilo Hart, al plantearse de ese modo, logra algunos beneficios teóricos, en particular porque ofrece facilidades para explicar los casos de indeterminación jurídica. Un caso de estos puede formularse del siguiente modo: la norma, p', no es jurídicamente válida y su negación, ᄀp', tampoco lo es; o bien, la proposición jurídica, p, no es verdadera y su negación, $\neg$ p, tampoco lo es. ${ }^{20}$ Según el positivismo jurídico estilo Hart, es lógicamente posible que ocurran casos de indeterminación jurídica al menos por dos razones:

1) Hart, por ejemplo, observó que dado que las reglas se expresan con términos clasificatorios generales, dejarán siempre una zona de penumbra, a la que llamó "textura abierta del lenguaje" y que actualmente puede ser entendida como vaguedad. ${ }^{21}$ Si se prohíbe la entrada de vehículos a un parque, la pregunta por la entrada de autos de juguete de propulsión eléctrica será un caso marginal y, por consiguiente, estará in-

19 Cfr. Raz, op. cit., The Authority ..., p. 58 (84).

20 Es decir: la proposición jurídica, p, no es verdadera ni falsa.

21 Cfr. Timothy Endicott, Vagueness in Law, Oxford, Oxford University Press, 2000, pp. 37 y 38 (73 y 74). 
determinada la solución jurídica al respecto. ${ }^{22}$ En este tipo de casos el juez habrá de tomar una decisión discrecional que, aunque no sea arbitraria ni irracional, nunca dejará de ser una elección. ${ }^{23}$

2) Aparte de la textura abierta del lenguaje o vaguedad, puede ocurrir (y con seguridad ocurre recurrentemente) que ninguna de dos normas contradictorias emane de los criterios que otorgan validez jurídica (o, en su caso, que ninguna de sus correspondientes proposiciones jurídicas lo haga). ${ }^{24}$ Por tomar un ejemplo prestado de Raz, en Inglaterra no hay una fuente para la proposición jurídica o regla de que está prohibido matar mariposas, pero tampoco la hay para la proposición jurídica o regla de que está permitido. ${ }^{25} \mathrm{Al}$ aplicar las tesis fundamentales del positivismo jurídico a dichos hechos negativos se llega, por modus tollens, a la conclusión de que la proposición jurídica de que está prohibido matar mariposas no es verdadera (o que la norma prohibitiva no es válida) y que la proposición jurídica que permite matar mariposas

22 Este tipo de casos apelan a la filosofía del lenguaje de tradición semántica. Para una explicación típica aplicada al positivismo jurídico estilo Hart véase David Brink, "Legal Interpretation, Objectivity, and Morality", en Brian Leiter (ed.), Objectivity in Law and Morals, Cambridge, Cambridge University Press, 2001, pp. 21 y 22.

23 Cfr. Hart, op. cit., cap. VII y p. 252 (28 del Post Scríptum al Concepto de Derecho).

24 Coleman así entiende una de las consecuencias del positivismo jurídico: "sólo un número determinado de estándares posee $C$ o $C_{1}$ de manera que puede surgir un caso en el que de acuerdo a la regla de reconocimiento no haya estándar jurídico alguno que sea idóneo o adecuado para su resolución" (Coleman, op. cit., p. 149, la traducción es mía); insinuándolo como un caso de laguna jurídica ( $c f r$. Idem, nota 9).

25 Cfr. Raz, op. cit., The Authority ..., p. 55 (80). Aunque Raz utiliza este ejemplo para rechazar una interpretación del positivismo jurídico, aquí lo utilizaré para ilustrar que cierta manera de interpretar el positivismo jurídico (acorde con la que estoy explorando) implica casos de indeterminación que surgen por el espacio lógico que permiten sus tesis fundamentales. 
tampoco lo es (o que la norma permisiva tampoco es válida); es decir, a una conclusión de indeterminación jurídica.

Obsérvese la importancia de introducir predicados como el de validez jurídica o el de verdad para evaluar normas o proposiciones jurídicas. Sin esos predicados (u otros que cumplan una función equiparable) no podríamos expresar con claridad un caso de indeterminación jurídica. Pensemos, por ejemplo, en el asunto de matar mariposas y tratemos de expresar que hay indeterminación jurídica pero sin esos predicados (o alguno semejante). Debido a esa restricción predicativa, sólo podríamos negar tanto la prohibición jurídica de matar mariposas como la permisión jurídica de hacerlo. Pero, como la negación de una prohibición es una permisión, se sigue que en realidad estamos expresando una contradicción jurídica, lo cual no es lo que queríamos expresar. Los predicados de validez jurídica y de verdad nos permiten expresar los casos de indeterminación jurídica sin confundirlos en absoluto con alguna contradicción jurídica.

Surge, sin embargo, el siguiente cuestionamiento: si el positivismo jurídico es una teoría que suministra las tesis fundamentales y los fundamentos de la validez jurídica de las normas o de la verdad de las proposiciones jurídicas, ¿a qué teoría entonces corresponde postular las tesis fundamentales y los fundamentos de las normas jurídicas o de las proposiciones jurídicas?

III. UNA INSUFICIENCIA DEL POSITIVISMO JURídICO ESTILO HART

He concluido el apartado anterior con una pregunta. Detallaré este cuestionamiento a través de nuestra intuición del concepto de respuesta jurídica correcta. Si las afirmaciones de validez jurídica de una norma o las de verdad de una proposición jurídica han de considerarse un tipo de respuestas jurídicas correctas, entonces, del mismo modo habría de decirse que las afirmaciones que niegan 
validez jurídica a una norma o verdad a una proposición jurídica pertenecen al mismo tipo de respuestas jurídicas; en cuyo caso, las afirmaciones de indeterminación jurídica serían igualmente respuestas jurídicas correctas. Pero esto conduce a una perplejidad debido a que tales afirmaciones de indeterminación jurídica justamente declaran que hay algún caso que no tiene una respuesta jurídica correcta. De modo que si llegamos a una conclusión de indeterminación jurídica cabe preguntarnos ¿cuál es el caso que carece de una respuesta jurídica correcta? Y, concomitante a ello, ¿de qué modo entonces se determinan las respuestas jurídicas correctas del tipo del que justamente no hay ninguna para ese caso?

Ilustraré estos cuestionamientos con un ejemplo. Supongamos que nos preguntamos si matar mariposas está jurídicamente prohibido. Las tesis fundamentales examinadas del positivismo jurídico nos indican que esta pregunta la debemos interpretar como un cuestionamiento acerca de la validez jurídica de una norma o bien acerca de la verdad de una proposición jurídica. Nuestras respuestas a estos cuestionamientos pueden ser: i) la prohibición de matar mariposas entendida como una norma es jurídicamente válida (o, entendida como una proposición jurídica, es verdadera) o ii) la prohibición de matar mariposas entendida como una norma no es jurídicamente válida (o, entendida como una proposición jurídica, no es verdadera). Si ahora nos preguntamos por la permisión jurídica de matar mariposas, las mismas tesis fundamentales examinadas autorizan dos respuestas: i) la permisión de matar mariposas entendida como una norma es jurídicamente válida (o, entendida como una proposición jurídica, es verdadera) o ii) la permisión de matar mariposas entendida como una norma no es jurídicamente válida (o, entendida como una proposición jurídica, no es verdadera). De esta manera, las tesis fundamentales examinadas pueden explicar la ocurrencia de que ni la prohibición de matar mariposas, ni su permisión, sean jurídicamente válidas (o, entendidas como proposiciones jurídicas, pueden explicar que ninguna sea verdadera). Supon- 
gamos que ocurre precisamente eso. Este tipo de respuesta, al estar suministrada por las tesis fundamentales del derecho examinadas, debe también considerarse como una respuesta jurídica correcta; pero, a su vez, lo que nos dice es que el derecho está indeterminado en algún punto. Entonces cabe preguntarnos, ¿cuál es el asunto que carece de respuesta jurídica correcta? ${ }^{26}$ No puede ser el asunto de la validez jurídica de la prohibición de matar mariposas, ni el de la permisión de hacerlo (ni tampoco el de la verdad de esa prohibición y esa permisión, entendidas como proposiciones jurídicas), pues ya hemos avisto que esos asuntos sí tienen respuestas jurídicas correctas. ¿Qué es lo que el positivismo jurídico está pasando por alto?

Eugenio Bulygin ha proporcionado una respuesta ante cuestionamientos análogos a los que he formulado. En su opinión, hay dos tipos de enunciados asociados con el derecho: i) enunciados sobre el derecho y ii) enunciados sobre el carácter deóntico de una acción según el derecho. Si lo entiendo bien, los enunciados del segundo tipo (que en adelante llamaré enunciados jurídicos ordinarios) ${ }^{27}$ se conforman simplemente de acciones evaluadas jurídicamente; por ejemplo: "Matar mariposas está jurídicamente prohibido". La acción considerada es matar mariposas y su carácter deóntico es el de prohibición jurídica. En cambio, un enunciado del primer tipo es aquel que predica verdad a un enunciado o proposición jurídica ordinaria. ${ }^{28}$ Considerando estos tipos de enunciados propone dos

26 Para ver algunas interrogantes cercanas a ésta véase Andrei Marmor, Positive Law and Objective Values, Oxford, Oxford University Press, 2001, pp. 142 y 143.

27 Entenderé que los enunciados jurídicos ordinarios significan, dependiendo de cómo se usen, una proposición jurídica ordinaria o una norma.

28 Entenderé que los enunciados sobre el derecho significan proposiciones sobre el derecho. Por otra parte, aunque Bulygin no lo mencione, consideraré que también son enunciados del primer tipo aquellos que predican validez jurídica a una norma; por ejemplo: "la prohibición de matar mariposas es jurídicamente válida". Este tipo de enunciados los considero equiparables a los enunciados que predican verdad de una proposición jurídica ordinaria porque en ambos casos sus negaciones lógicas no atribuyen ningún carácter deóntico jurídico a la acción 
conceptos de determinación. Por un lado, hay determinación de los enunciados sobre el derecho y, por otro lado, lo que Bulygin llama la determinación del "resultado", es decir, "la determinación de la solución que el derecho estipula para un cierto problema"29 o caracterización deóntica unívoca de una acción. De estas observaciones podemos inferir una plausible respuesta que Bulygin podría proporcionar a los cuestionamientos que señalo. Tenemos que distinguir entre las tesis que determinan las proposiciones sobre el derecho de las tesis que determinan las normas o, en su caso, las proposiciones jurídicas ordinarias. Si las distinguimos tiene sentido decir que según las tesis fundamentales examinadas del positivismo jurídico se puede llegar a una conclusión de indeterminación (una proposición sobre el derecho) y, a su vez, explicar cuál es la cuestión que carece de respuesta jurídica correcta. Consideremos nuevamente el ejemplo de las regulaciones sobre matar mariposas. Si al aplicar las tesis fundamentales examinadas llegamos a la conclusión de que no es verdadero que esté jurídicamente prohibido matar mariposas y tampoco es verdadero que esté jurídicamente permitido matar mariposas (una proposición sobre el derecho, en particular, de indeterminación jurídica), aún queda espacio para mantener cuál es el caso que carece de respuesta jurídica correcta; a saber: el caso de si matar mariposas está jurídicamente prohibido

mencionada en el enunciado que niegan. En cambio, los enunciados jurídicos ordinarios, aunque tengan una formulación cercana a los enunciados que predican validez jurídica a una norma, son en mi opinión distintos, porque sus negaciones lógicas sí atribuyen un carácter deóntico jurídico a la acción sobre la que tratan; negar, por ejemplo, que esté jurídicamente prohibido matar mariposas significa la afirmación de que está permitido hacerlo. De no haber comprendido adecuadamente a Bulygin, entonces su distinción no ayudaría a resolver el problema que señalo en la parte II del trabajo; pero sí lo podría hacer la distinción que equivocadamente estoy suponiendo como suya.

29 Eugenio Bulygin, "Objectivity of Law in the View of Legal Positivism", en Paolo Comanducci y Ricardo Guastini (eds.), Analisi e Diritto, Torino, Giappichelli, 2004, p. 224, la traducción es mía. 
o no (sea que lo entendamos como un cuestionamiento por una norma o por una proposición jurídica ordinaria). Mismo que puede reformularse como un caso sobre si matar mariposas está jurídicamente prohibido o permitido. ${ }^{30}$

Nótese que un asunto es el caso sobre si es verdadero que matar mariposas está jurídicamente prohibido o no; y, otro distinto, el caso sobre si matar mariposas está jurídicamente prohibido o permitido. Las posibles respuestas al primer caso son: a) es verdadero que matar mariposas está jurídicamente prohibido o b) no es verdadero que matar mariposas esté jurídicamente prohibido. En cambio, las posibles respuestas al segundo caso son: a) matar mariposas está jurídicamente prohibido o b) matar mariposas está jurídicamente permitido. Esta distinción es importante, pues mientras es consistente afirmar que no es verdadero que matar mariposas esté jurídicamente prohibido y, a su vez, que tampoco lo es que matar mariposas esté jurídicamente permitido; no es consistente, en cambio, afirmar que matar mariposas está jurídicamente prohibido y, a su vez, jurídicamente permitido. Cuando agregamos el predicado "es verdadero" a una proposición jurídica ordinaria (o cuando agregamos el predicado "es jurídicamente válida" a una norma) abrimos un espacio lógico que nos permite afirmar con consistencia que ciertos casos están indeterminados. Sin esos predicados, el espacio se cancela. Esto nos muestra una dimensión en la que podemos considerar los casos jurídicos, no como cuestiones sobre verdad o validez jurídica, sino como cuestiones del carácter deóntico jurídico que ciertas acciones tienen. El positivismo jurídico ha estado demasiado enfocado en proveer las tesis fundamentales de las proposiciones sobre el derecho; a saber, sobre qué justifica aplicar el predicado "es verdadero" a una proposición jurídica ordinaria o el predicado "es jurídicamente válida" a una norma, pero no nos ha dicho lo suficiente sobre las tesis fundamentales de las normas jurídicas o, en

зо Esto debido a que la negación de una prohibición es una permisión. 
su caso, de las proposiciones jurídicas ordinarias; es decir, sobre qué justifica que ciertas acciones tengan un carácter deóntico unívoco.

iv. El fracaso de dos estrategias de SOlución

A continuación se considerará dos posibles soluciones que el positivismo jurídico estilo Hart podría proveer para este problema. Sin embargo, se observará que ninguna es satisfactoria.

\section{LA ESTRATEgIA POR SIMPLE ELIMINACIÓN DE LOS PREDICADOS “ES VERDADERO” O “ES JURÍDICAMENTE VÁLIDA"}

Tal vez la primera solución que alguien se podría imaginar es postular tesis fundamentales similares a las examinadas, pero ahora para determinar los caracteres deóntico jurídicos que corresponden a las acciones; es decir, para determinar las normas jurídicas o las proposiciones jurídicas ordinarias. Piénsese, por ejemplo, en el planteamiento de Coleman. Sin tener que rechazar T1 de Coleman, podría agregarse la siguiente:

T2 de Coleman:

Una acción es jurídicamente obligatoria, prohibida o permitida en una determinada comunidad, M, si y sólo si la norma correspondiente posee la característica $\mathrm{C}$ o la característica $\mathrm{C}_{1}$, según lo estipule su propia regla de econocimiento.

Esta tesis es problemática porque implica que al considerar una norma cualquiera y su negación, necesariamente alguna estará respaldada por algún fundamento del derecho. Si, por ejemplo, la norma que prohíbe matar mariposas no posee ni la característica $C$ ni la $C_{1}$, entonces, por modus tollens, se seguirá la negación de la versión jurídica de dicha norma; es decir, que matar mariposas está 
jurídicamente permitido y, por consiguiente, que esta última norma posee alguna de las características $C_{\mathrm{o}} C_{1}$. Sin embargo, es perfectamente posible que una norma y su negación no posean ningún fundamento jurídico que las respalde. ${ }^{31}$

El mismo problema se presenta con una tesis complementaria al Ensayo de Raz. Supongamos que ésta fuera la siguiente:

Tesis complementaria al Ensayo de Raz:

$\mathrm{S}(\mathrm{p})$ es una condición lógicamente necesaria y suficiente para $\mathrm{p}$.

$\mathrm{S}(\neg \mathrm{p})$ es una condición lógicamente necesaria y suficiente para $\neg$ p.

Si p no tiene una fuente que la respalde, se seguirá $\neg p$, y de ésta última proposición, por modus ponens, se derivará que $\mathrm{S}(\neg \mathrm{p})$. Lo cual expone que al considerar una proposición normativa cualquiera y su negación, necesariamente alguna estará respaldada por una fuente. Pero como se ha señalado, es perfectamente posible $\neg \mathrm{S}(\mathrm{p})$ y $\neg \mathrm{S}(\neg \mathrm{p})$.

\section{La estrategia de Raz}

La segunda solución fue ideada por Raz y aunque su exposición es algo compleja, vale la pena considerarla de principio a fin. $\mathrm{Su}$ propuesta parte de la premisa de que existen razones jurídicas concluyentes que dependen en su totalidad de fuentes (de fuentes que proveen razones jurídicas y de fuentes que señalan cuáles son concluyentes y cuáles no). Y, por otra parte, adopta la premisa de que también existen permisiones concluyentes, pero que ellas nunca de-

31 Críticas asociadas con está han sido expuestas por Raz y Dworkin ( $c f r$. Raz, op. cit., The Authority ..., pp. 54 y 55 (79 y 80); y, Ronald Dworkin, A Matter of Principle, Cambridge, Massachusetts, Harvard University Press, 1985, pp. 131-134 (174-177). 
penden de fuentes, más bien dependen de que no haya razones jurídicas concluyentes a favor de una acción u omisión. ${ }^{32}$ Por ejemplo, si queremos averiguar cuál es el carácter deóntico jurídico de la acción de matar mariposas, debemos preguntarnos si existen razones jurídicas concluyentes para realizarla o para no realizarla. Si esas razones jurídicas concluyentes para realizarla no existen, entonces no realizar la acción estará permitido jurídica y concluyentemente; si, a su vez, tampoco existen razones jurídicas concluyentes para no realizarla, entonces la acción estará permitida jurídica y concluyentemente (como consecuencia, la acción sería facultativa jurídica y concluyentemente).

En ese orden de ideas, las tesis fundamentales del derecho que finalmente propuso Raz fueron las siguientes:

T1 de Raz

$$
\text { (马s) } \mathrm{s} \operatorname{LR}_{\mathrm{c}} \mathrm{x}, \phi \leftrightarrow \mathrm{LR}_{\mathrm{c}} \mathrm{x}, \Phi^{33}
$$

Donde ( $\left.\mathrm{H}_{\mathrm{s}}\right) \mathrm{s} \mathrm{LR}_{\mathrm{c}} \mathrm{x}, \Phi$ significa "Existe una $s$ tal que $s$ es una razón jurídica concluyente para que $x$ haga $\phi ”$. Tesis a la que agregó (o de la que derivó, con apoyo de lógica deóntica estándar):

T2 de Raz

$$
\sim \operatorname{LR}_{\mathrm{c}} \mathrm{x}, \Phi \leftrightarrow \operatorname{LPer}_{\mathrm{c}} \mathrm{x}, \sim \Phi^{34}
$$

32 En ese sentido se aparta de la tesis de que la determinación del carácter deóntico jurídico de una acción sólo se deriva de presencia de una fuente (las permisiones concluyentes están determinadas por la falta de fuentes). Esto nos recuerda la propuesta de Hans Kelsen de que las regulaciones de un orden jurídico no sólo son positivas; sino también negativas. Kelsen afirmó que si una conducta no estaba prohibida, entonces estaba permitida de un modo negativo. Dicha permisión funcionaba como guía de conducta para un juez, por ejemplo, para absolver o rechazar una demanda (cfr. Kelsen, Teoría Pura del Derecho, trad. Roberto J. Vernengo, Ciudad de México, Porrúa, 1991, pp. 28-30 y 251-258).

${ }^{33}$ Obtenida por analogía de Raz, op. cit., The Authority ..., p. 66 (92). Con esta tesis Raz está postulando una equivalencia entre lo que en mi opinión son proposiciones jurídicas de existencia (un tipo de proposiciones sobre el derecho) y proposiciones jurídicas ordinarias. Equivalencia que, en mi opinión, es el aspecto central de su propuesta.

${ }^{34}$ Raz, op. cit., The Authority ..., p. 76 (105). 
Si a T1 de Raz aplicamos una transposición y a la fórmula resultante junto con T2 de Raz aplicamos un silogismo hipotético, se sigue:

T3 de Raz

$$
\sim\left(\mathrm{H}_{\mathrm{s}}\right) \mathrm{s}_{\mathrm{c}} \mathrm{LR}, \Phi \leftrightarrow \operatorname{LPer}_{\mathrm{c}} \mathrm{x}, \sim \phi
$$

Lo que expresa el sentido en el que para Raz las permisiones jurídicas concluyentes nunca dependen de fuentes. Hasta aquí todo bien. ${ }^{35}$ Pero parece que no hay espacio para ningún tipo de insuficiencia jurídica, pues o bien existirá una s tal que sea una razón jurídica concluyente para que x haga $\Phi$ o bien no existirá. De modo que o bien se requerirá concluyentemente que $\mathrm{x}$ haga $\Phi$ o bien se permitirá concluyentemente que no lo haga. Aunque esto evade el problema de la primera estrategia de que siempre existirá una fuente que respalde una proposición normativa ordinaria o su negación, ${ }^{36}$ aún no explica cómo son posibles los casos de indeterminación jurídica.

La respuesta anticipada de Raz fue que su propuesta no eliminaba la posibilidad de los casos de indeterminación jurídica, pero que

35 Jorge Rodríguez y Pablo Navarro en coautoría reconstruyen en términos parecidos la propuesta de Raz y le ven problemas a sus pretensiones. Expresan T2 de Raz de la siguiente manera: “ $\neg \mathrm{LRp} \leftrightarrow \mathrm{L} \neg \mathrm{Rp}$ ” y la interpretan, primero, como una tesis cuyos componentes son proposiciones de pertenencia a un sistema jurídico, haciendo la observación de que no es una verdad lógica como Raz afirma (cfr. Pablo Navarro y Jorge Rodríguez, Deontic Logic and Legal Systems, Nueva York, Cambridge University Press, 2014, pp. 163 y 164). Enseguida la interpretan como una tesis cuyos componentes son proposiciones de verdad de proposiciones de pertenencia a un sistema jurídico; en este caso, concluyen que lo que Raz afirma sí es una verdad lógica, pero que finalmente "no dice nada sobre la posibilidad de que ni $O p$ ni $\neg O p$ pertenezcan a un sistema jurídico, de manera que $p$ no esté regulada por el de derecho" (Ibidem, p. 166, la traducción es mía). En mi opinión, la primera interpretación de estos autores es correcta, pero están equivocados en que la fórmula de Raz no sea una verdad lógica; lo es una vez que consideramos T1 de Raz (una fórmula que pasan por alto estos autores).

36 Lo evade porque, como se ha visto, para Raz las permisiones jurídicas concluyentes no están fundadas en fuentes, de modo que su propuesta no implica que una proposición normativa ordinaria o su negación forzosamente estén respaldadas por una fuente jurídica. 
ésta era producto de la indeterminación de las fuentes que proveen razones jurídicas concluyentes. Es decir, podría estar indeterminado si hay o no una fuente que sea una razón jurídica concluyente a favor de realizar cierta acción. Sus señalamientos se dirigen a dos grupos de casos: 1) cuando los hechos que son razones jurídicas están indeterminados, por vaguedad, textura abierta o cualquier otro factor; ${ }^{37} \mathrm{y}, 2$ ) cuando hay conflictos de razones no resueltos, entre los cuales encuentra dos subcasos relevantes: a) razones que no pueden competir en absoluto; ${ }^{38} \mathrm{y}, \mathrm{b}$ ) consideraciones no basadas en fuentes que determinan la validez o no de lo que prima facie es derecho. ${ }^{39}$

En todos los casos anteriores, Raz, para hablar de indeterminación (así como de lagunas y completitud de un sistema jurídico), introduce lo que en este artículo se ha llamado proposiciones sobre el derecho. Asume que la indeterminación trata de casos donde las proposiciones jurídicas ordinarias no son verdaderas ni falsas y creo que esto no nos lo podríamos explicar sin que agreguemos tesis adicionales como las siguientes:

T1' de Raz

T2' de Raz (马s) $\mathrm{s} \mathrm{LR}_{\mathrm{c}} \mathrm{x}, \phi$ es verdadera $\leftrightarrow \mathrm{LR}_{\mathrm{c}} \mathrm{x}, \phi$ es verdadera

$\sim \operatorname{LR}_{\mathrm{c}} \mathrm{x}, \Phi$ es verdadera $\leftrightarrow \operatorname{LPer}_{\mathrm{c}} \mathrm{x}, \sim \Phi$ es verdadera ${ }^{40}$

37 Raz, op. cit., The Authority ..., p. 72 (100).

38 Ibidem, p. 75 (103).

39 Idem.

40 En un trabajo especializado Juan Moreso, Pablo Navarro y Cristina Redondo, en coautoría, analizan varias tesis de Raz con operadores de verdad. Ellos llegan a la conclusión de que una equivalencia que propone Raz entre $\sim \operatorname{LR}_{c} \mathrm{x}, \Phi \mathrm{y}$ $\mathrm{L} \sim \mathrm{R}_{\mathrm{c}} \mathrm{x}, \Phi$, puede ser expresada con el operador de la verdad del siguiente modo: $\mathrm{T} \sim \mathrm{LR}_{\mathrm{c}} \mathrm{x}, \phi \leftrightarrow \mathrm{TL} \sim \mathrm{R}_{\mathrm{c}} \mathrm{x}, \phi$, donde T significa "es verdadero que" (cfr. Juan Moreso et al., "Sobre la Lógica de las Lagunas en el Derecho", en Crítica: Revista Hispanoamericana de Filosofia, vol. 33, n 99, 2001, pp. 63-67). Siguiendo las explicaciones de 
Una cuestión adicional sobre la que Raz no entra en mucho detalle es la de cuáles son las tesis básicas que nos autorizarían a inferir que (马s) s LR x, $\Phi$ no es verdadera ni falsa. Eso es algo que, para los presentes propósitos, podemos pasar por alto. Lo importante aquí es que la indeterminación es posible no porque falte cierta fuente; sino porque no es verdadero ni falso que exista cierta fuente. El Ensayo de Raz que vimos en la parte II muestra que uno de los proveedores de indeterminación jurídica era la ausencia de fuentes; ahora, en esta nueva propuesta, ese proveedor está desactivado: según T1 y T2 de Raz, que falte cierta fuente conduce a una permisión (concluyente); y, según T1'y T2' de Raz que sea verdadero que falta cierta fuente conduce a que es verdadero un enunciado jurídico ordinario permisivo. En ninguno de estos dos casos se presenta indeterminación alguna.

Sin duda es una propuesta admirable. Para evadir que la ausencia de fundamentos del derecho que determinan el carácter deóntico jurídico de una acción fuera imposible, Raz excluyó a las permisiones jurídicas concluyentes de la tesis de las fuentes. Pero como eso no explicaba la indeterminación jurídica, porque ahora sería un enigma de dónde surgía y además se requería el concepto de verdad para explicarla; entonces agregó que también las fuentes podrían estar indeterminadas e insinuó las tesis adicionales 1'y 2' arriba especificadas donde se introducen proposiciones sobre el derecho.

Sólo surge una extrañeza con estas tesis fundamentales: parece contradecir la intuición del positivismo jurídico de que el derecho puede "callar". Aunque Raz sólo dice que: "no hay lagunas cuando el derecho es mudo", ${ }^{41}$ es de suponerse que en realidad quiso decir que el derecho ya no puede simplemente enmudecer, dado que los permisos concluyentes producto de la falta de fuentes apropiadas

estos autores, dado que $\sim \operatorname{LR}_{\mathrm{c}} \mathrm{x}, \phi \leftrightarrow \operatorname{LPer}_{\mathrm{c}} \mathrm{x}, \sim \Phi$, todo indica que ellos no tendrían inconveniente en admitir $\mathrm{T} \sim \mathrm{LR}_{\mathrm{c}} \mathrm{x}, \phi \leftrightarrow \operatorname{TLPer}_{\mathrm{c}} \mathrm{x}, \sim \phi$, que es justamente lo que propongo como T2' de Raz.

41 Raz, op. cit., The Authority ..., p. 77 (105). 
también son jurídicos. ${ }^{42}$ Las únicas lagunas que pueden ser el caso son aquellas que surgen cuando el derecho "habla con voz incierta (simple indeterminación) o habla con muchas voces (conflictos no resueltos)". ${ }^{43}$ Con ello Raz rechaza una buena intuición del positivismo jurídico, pero a cambio nos pide que consideremos la ventaja que supone su propuesta: se explica la intuición jurídica de que lo que no está prohibido está permitido; ${ }^{44}$ pues si aceptamos su propuesta dicha intuición sería una verdad analítica.

No obstante la meticulosidad de esta propuesta, conduce a un problema. Éste se deriva de la, a veces olvidada, discreción afirmativa o explícita. Ésta consiste en una facultad concedida, por

42 John Gardner parece concordar con Raz en gran medida: "Cuando aquí hablo de 'lagunas', no quiero decir que el derecho guarda silencio en algunos casos. Las reglas de clausura (tales como: 'todo lo que no está prohibido por el derecho, está permitido por el derecho’) son perfectamente aptas para evitar el silencio jurídico" (Gardner, op. cit., pp. 34 y 35, la traducción es mía). Es destacable además que la regla de clausura que menciona es en su opinión una ley de la lógica deóntica ( $c f r$. Idem, nota 29).

43 Raz, op. cit., The Authority ..., p. 77 (105).

44 El enunciado "lo que no está prohibido, está permitido" puede interpretarse de dos modos: como una ley de la lógica deóntica estándar que indica que la negación de una prohibición implica materialmente una permisión; o, como la afirmación de que la falta de una prohibición implica materialmente una permisión. La primera interpretación es inútil como regla de clausura jurídica, ya que lo que está en cuestión cuando se habla de lagunas jurídicas es lo que sucede cuando falta una prohibición y la lógica deóntica estándar no proporciona ninguna indicación al respecto. La segunda interpretación es más interesante porque surge la pregunta sobre la contingencia o necesidad de su verdad. Raz parece inclinarse por esta interpretación y, en consecuencia, nos está sugiriendo que dicha afirmación es una verdad analítica, incluso tal vez un candidato para ley de algún tipo de lógica deóntica. Gardner, por su parte, no necesariamente ha de ser considerado como partidario de la primera interpretación ya que sólo dice que se trata de una ley de la lógica deóntica sin aclarar si es de la lógica deóntica estándar o de alguna otra; si fuera esto último, Gardner estaría desarrollando la postura de Raz. Vale la pena recordar que Kelsen fue defensor de la afirmación de que lo que no está prohibido está permitido, sugerentemente bajo esta segunda interpretación ( $c f r$. Kelsen, op. cit., pp. 28-30 y 251-258). 
ejemplo, a un juez para que a su elección decida de un modo u otro cierto caso. ${ }^{45}$ Podemos imaginarnos el caso hipotético donde un juez tiene discreción afirmativa para asegurar el cumplimiento de una obligación alimentaria. Supongamos que su discreción radica en solicitarle al deudor un depósito o bien su afianzamiento. Este tipo de discreción supone que las partes involucradas no tienen derechos ni obligaciones al respecto. No se puede decir que el deudor tenga la obligación de hacer un depósito, porque de tenerla, contradiríamos la hipótesis de la facultad judicial; lo mismo ocurriría si se afirma que el deudor tiene la obligación de afianzarse. Y, si se desea investigar, por el lado de los derechos del acreedor alimentario, tampoco se puede decir que tenga algún derecho a un modo específico de obtener una garantía, nuevamente porque de hacerlo contradiríamos la hipótesis de la facultad judicial. De esta manera la discreción afirmativa sobre el caso de los derechos y obligaciones del juez al resolver un caso de garantía alimentaria, implica indeterminación jurídica sobre el caso de los derechos y obligaciones de las partes involucradas respecto al tipo específico de garantía que ha de satisfacerse. Lo interesante de casos como este es que la ausencia de derecho parece deberse a un enmudecimiento de éste; que es concomitante a la discreción afirmativa que tiene el juez y no a una "voz incierta" o "multiplicidad de voces" del derecho.

El mismo problema puede verse desde otro punto de vista. Supóngase el mismo caso del deudor alimentario. Ahora aplíquense las tesis fundamentales del derecho de Raz a la cuestión sobre si el

45 Aunque se encuentra muy difundida la distinción de Dworkin entre i) discreción débil como tener la decisión final de un caso; ii) discreción débil como tener que usar el discernimiento para resolver un caso; y, iii) discreción fuerte por default donde no hay estándares vinculantes para el decisor (pero tampoco una facultad); se ha olvidado que Dworkin en algún momento se refirió a la discreción afirmativa, al parecer, considerando que sus características distintivas son que surge de una facultad explícita y no se dice que, al utilizarla, los jueces hagan derecho ( $c f r$. Ronald Dworkin, "Judicial Discretion", en The Fournal of Philosophy, $\mathrm{n}^{\circ} 60,1963$, p. 634, n. 6). 
deudor tiene o no una razón jurídica concluyente a favor de hacer el depósito. No puede haberla, pues de existir contradiríamos la hipótesis de que el juzgador tiene discreción afirmativa. Ahora aplíquense nuevamente esas mismas tesis fundamentales a la cuestión sobre si el deudor tiene o no una razón jurídica concluyente a favor de no hacer el depósito. Tampoco puede haberla, porque de existir también contradiríamos la hipótesis de discreción afirmativa. De ello se sigue que el deudor tiene los permisos jurídicos concluyentes de hacer el depósito y de no hacerlo; lo cual equivale a una facultad jurídica para el deudor alimentario. Pero si el deudor está facultado a hacer el depósito, ¿cómo es posible que el juzgador tenga discreción entre exigir un depósito y exigir un afianzamiento? Para salir de este apuro Raz tendría que decir que el derecho de algún modo ha hablado con "voz incierta" o "multiplicidad de voces" sobre los derechos y obligaciones del deudor en lo que respecta al aseguramiento de su deuda, pero no se ve de dónde pueda sostenerse tal afirmación.

La discreción afirmativa muestra, en consecuencia, que no deja de tener sentido el concepto de indeterminación jurídica por silencio jurídico y evidencia que las tesis fundamentales de Raz, al no dar lugar a ello, no pueden ser correctas.

\section{Proposiciones sobre el derecho y Lógica de la verdad}

En las partes precedentes del trabajo se ha expuesto que el positivismo jurídico estilo Hart tiene un problema en su planteamiento básico: sólo expone las tesis fundamentales para las proposiciones sobre el derecho y eso sólo logra explicar los fundamentos de dichas proposiciones. Pero, tal vez, esto no es del todo cierto. Resulta plausible pensar que las tesis fundamentales de las proposiciones sobre el derecho implican tesis fundamentales de las proposiciones jurídicas ordinarias. No parecería que alguien fuera coherente si afirma que es verdadero que está jurídicamente prohibido matar mariposas, 
pero que no está jurídicamente prohibido matar mariposas; esto indica que hay una relación lógica de implicación material entre las proposiciones sobre el derecho y las proposiciones jurídicas ordinarias. Georg Henrik von Wright admitió la posibilidad de esta relación de implicación pocos años después de elaborar sus primeras lógicas de la verdad (truth-logics). ${ }^{46}$ Dichas lógicas incorporan el operador de la verdad, en adelante T, que significa "es verdadero que" y, una de esas lógicas, llamada TLM, entre otros axiomas postula el siguiente:

$$
\text { A6. } \quad \mathrm{Tp} \rightarrow \mathrm{p}^{47}
$$

Que se lee del siguiente modo: "si es verdadero p, entonces p", y expresa la relación de implicación material que he anunciado. Si aceptamos esta plausible relación lógica, entonces al positivista jurídico estilo Hart le bastaría con postular tesis fundamentales de las proposiciones sobre el derecho para implicar tesis fundamentales de las proposiciones jurídicas ordinarias. Retomemos el Ensayo de Raz. Recordemos que éste afirma que el hecho de que una proposición jurídica (ordinaria), p, emane de una fuente apropiada es condición necesaria y suficiente para que p sea verdadera. Esto lo podemos simbolizar del modo siguiente:

\section{Ensayo de Raz: $\quad \mathrm{Sp} \leftrightarrow \mathrm{Tp}$}

Donde S significa "tiene una fuente" y T es el operador de verdad (que significa "es verdadero que"). Al considerar la fórmula del Ensayo de Raz y A6 de TLM podemos llegar, por silogismo hipotético, a la conclusión de que el hecho de que una proposición jurídica ordinaria, p, tenga una fuente es condición suficiente para

46 Para consultar sus primeras lógicas de la verdad véase Georg H. von Wright, Truth, Knowledge and Modality, Oxford, Basil Blackwell, 1984, pp. 26-36. Para consultar la introducción de esa relación de implicación véase ibidem, "Truth-logics", en Logique et Analyse, no 30, 1987, pp. 311 y 313.

47 Ibidem, p. 322. 
que p. Es decir, a la tesis: $\mathrm{Sp} \rightarrow$ p. La cual puede considerarse una (o la) tesis fundamental de las proposiciones jurídicas ordinarias. De modo que después de todo el positivismo jurídico estilo Hart, al menos en la versión que interpreta sus tesis fundamentales con pretensiones de determinar la verdad de las proposiciones jurídicas ordinarias, plausiblemente implica una tesis fundamental para las proposiciones jurídicas ordinarias; precisamente la tesis que en la parte IV del artículo reclamaba por su ausencia.

Aunque esto puede sonar como un argumento favorable para ese tipo de positivismo jurídico, más bien funcionará como una objeción a los fundamentos del derecho que postula. Ahora que se ha visto que no sólo tiene sentido indicar las condiciones que determinan cuándo una norma adquiere validez jurídica, o bien, cuándo una proposición jurídica es verdadera, sino que también lo tiene señalar las condiciones que determinan el carácter deóntico jurídico de las acciones (es decir, la determinación de las proposiciones jurídicas ordinarias), se ha de reconocer que los fundamentos del derecho pueden ser entendidos más ampliamente como las condiciones que determinan ambas cuestiones.

El positivismo jurídico estilo Hart asume que existe la posibilidad de un sistema jurídico que señala, como esas condiciones (es decir, como fundamentos del derecho), a hechos sociales (legislación, decisiones judiciales pasadas, etc.). ${ }^{48} \mathrm{Y}$ esa posibilidad es todo lo que se necesita para mostrar que ese tipo de positivismo jurídico asume un grupo de afirmaciones patentemente falsas. Veamos:

Según lo que he dicho en esta parte del trabajo, el positivismo jurídico estilo Hart, al menos en la versión que interpreta sus tesis fundamentales con pretensiones de determinar la verdad de las proposiciones jurídicas ordinarias, supone dos tesis fundamentales. Para abreviar, recurriré a las versiones simbólicas antes expuestas:

48 Esta afirmación es tan débil que constituye una tesis común para el positivismo jurídico incluyente y el positivismo jurídico excluyente. 


\section{T1 PJ: $\quad \mathrm{Sp} \leftrightarrow \mathrm{Tp}$ \\ T2 PJ: $\quad \mathrm{Sp} \rightarrow \mathrm{p}$}

Debemos advertir que una instancia de T2 PJ es:

$$
\text { T3 PJ: } \quad \mathrm{S} \neg \mathrm{p} \rightarrow \neg \mathrm{p}
$$

\section{De T2 PJ y T3 PJ se sigue:}

$$
\text { T4 PJ: } \quad(\mathrm{Sp} \& \mathrm{~S} \neg \mathrm{p}) \rightarrow(\mathrm{p} \& \neg \mathrm{p})^{49}
$$

Pero, dado que una tesis de lógica clásica es que: $\neg(\mathrm{p} \& \neg \mathrm{p})$, de T4 PJ y dicha tesis de lógica clásica se deriva, por modus tollens, que:

$$
\text { T5 PJ: } \quad \neg(\text { Sp \& S } \neg \text { p) }
$$

Sin embargo, T5 PJ es evidentemente falsa: puede ocurrir que tanto p como $\neg$ p tengan una fuente. Visto de otro modo: T5 PJ niega la posibilidad de un sistema jurídico que señale como fundamentos del derecho a hechos sociales y, a su vez, que de estos emanen proposiciones jurídicas ordinarias contradictorias; en tanto que no hay nada en ninguno de los conceptos de las fuentes del derecho (legislación, decisiones judiciales pasadas, etc.) que cancele esa posibilidad. ${ }^{50}$ Esto muestra que de ser verdadera T5 PJ, los fundamentos del derecho tienen que ser tales que su concepto evite la posibilidad de contradicciones jurídicas y, por consiguiente, que

49 Esto puede demostrarse mediante una prueba condicional: se comienza por suponer Sp \& S $\neg$ p. Se aplican dos eliminaciones de la conjunción para aislar tanto a Sp como a $\mathrm{S} \neg$ p. Estas dos afirmaciones junto con T2 PJ y T3 PJ, implican, por modus pones, p y $\neg$ p. Por introducción de la conjunción se sigue: p \& $\neg$ p.

50 El concepto de legislación, por ejemplo, no implica que es lógicamente imposible legislar p y $\neg$ p. El positivismo jurídico estilo Hart al implicar la conclusión que todos los conceptos de las fuentes sociales contienen ese tipo de imposibilidades lógicas, contiene una serie de afirmaciones patentemente falsas. 
el positivismo jurídico estilo Hart tiene que modificar o en su caso precisar aún más cuáles son los fundamentos del derecho.

\section{vi. Conclusiones}

La parte III del trabajo expone una insuficiencia en el positivismo jurídico estilo Hart, a saber, que no proporciona las tesis fundamentales de las proposiciones jurídicas ordinarias. La parte IV expone dos estrategias para cumplir con esa tarea, pero, según se argumenta, ambas fracasan. Y, la parte V, trata con una estrategia más, encontrando que contradice la suposición del positivismo jurídico estilo Hart de que es posible un sistema jurídico cuyos fundamentos sean fuentes sociales. De estas conclusiones se puede extraer un dilema para los positivistas jurídicos estilo Hart: o reconocen que su teoría está incompleta por carecer de tesis fundamentales para las proposiciones jurídicas ordinarias o, al aceptar las tesis fundamentales que con ayuda del axioma $\mathrm{Tp} \rightarrow \mathrm{p}$ se derivan, admiten que los fundamentos del derecho que proponen no pueden ser sin más especificaciones las fuentes sociales que tradicionalmente han defendido (legislación, decisiones judiciales pasadas, etc.).

\section{Fuentes}

\section{Bibliografía}

BRINK, David, "Legal Interpretation, Objectivity, and Morality", en Brian Leiter (ed.), Objectivity in Law and Morals, Cambridge, Cambridge University Press, 2001, pp. 12-65.

Dworkin, Ronald, A Matter of Principle, Cambridge, Massachusetts, Harvard University Press, 1985. (Versión en español: Una Cuestión de Principios, trad. Victoria Boschiroli, Buenos Aires, Siglo XXI, 2012). 
- Law's Empire, Cambridge, Massachusetts, Harvard University Press, 1986. (Versión en español: El Imperio de la fusticia, 2ª edición, trad. Claudia Ferrari, Barcelona, Gedisa, 1992).

EndicotT, Timothy, Vagueness in Law, Oxford, Oxford University Press, 2000. (Versión en español: La Vaguedad en el Derecho, trads. Alberto del Real y Juan Vega, Madrid, Dykinson, 2006).

Gardner, John, Law as a Leap of Faith: Essays on Law in General, Oxford, Oxford University Press, 2012.

Hart, H. L. A., The Concept of Law, 2a. edición, Oxford, Oxford University Press, 1994. (Versión en español: El Concepto de Derecho, 2a. edición, trad. Genaro Carrió, Buenos Aires, Abeledo-Perrot, 1995 y Post Scríptum al Concepto de Derecho, trad. Rolando Tamayo, Ciudad de México, UNAM, 2000).

Himma, Keneth, "Inclusive Legal Positivism", en Jules Coleman y Scott Shapiro (eds.), Oxford Handbook of Jurisprudence and Philosophy of Law, Oxford, Oxford University Press, 2002, pp. 125-165. (Versión en español: "Positivismo Jurídico Incluyente", trad. Pedro Villarreal, Problema: Anuario de Filosofia y Teoría del Derecho, nº 8, 2014, pp. 353-430).

Kelsen, Hans, Teoría Pura del Derecho, trad. Roberto J. Vernengo, Ciudad de México, Porrúa, 1991.

Marmor, Andrei, Positive Law and Objective Values, Oxford, Oxford University Press, 2001.

—., Philosophy of Law, Princeton, Princeton University Press, 2011.

Navarro, Pablo y Jorge Rodríguez, Deontic Logic and Legal Systems, Nueva York, Cambridge University Press, 2014.

Patterson, Dennis, Law and Truth, Oxford, Oxford University Press, 1996.

Raz, Joseph, The Authority of Law: Essays on Law and Morality, Oxford, Oxford University Press, 1979. (Versión en español: La Autoridad del Derecho. Ensayos sobre Derecho y Moral, trad. Rolando Tamayo, Ciudad de México, Ediciones Coyoacán, 2011).

Shapiro, Scott, Legality, Cambridge, Massachusetts, Harvard University Press, 2011. (Versión en español: Legalidad, trad. Diego Papayannis y Lorena Ramírez, Madrid, Marcial Pons, 2014). 
Von Wright, Georg H., Truth, Knowledge and Modality, Oxford, Basil Blackwell, 1984.

Waluchow, Wilfrid, Inclusive Legal Positivism, Oxford, Oxford University Press, 1994. (Versión en español: Positivismo Furídico Incluyente, trad. Marcela Gil y Romina Tesone, Madrid, Marcial Pons, 2007).

2. Hemerografía

Bulygin, Eugenio, "Objectivity of Law in the View of Legal Positivism", en Paolo Comanducci y Ricardo Guastini (eds.), Analisi e Diritto, Torino, Giappichelli, 2004, pp. 219-227.

Coleman, Jules, "Negative and Positive Positivism", en The Journal of Legal Studies, vol. 11, n 1, 1982, pp. 139-164.

Dworkin, Ronald, "Judicial Discretion", en The Gournal of Philosophy, ${ }^{\circ}$ 60, 1963, pp. 624-638.

Moreso, Juan et al., "Sobre la Lógica de las Lagunas en el Derecho", en Crítica: Revista Hispanoamericana de Filosofía, vol. 33, n 99, 2001, pp. 47-73.

RAz, Joseph, “H. L. A. Hart (1907-1992)”, en Utilitas, vol. 5, nº 2, 1993, pp. 145-156.

ToH, Kevin, "Hart's Expressivism and his Benthamite Project", en Legal Theory, $\mathrm{n}^{\circ}$ 11, 2005, pp. 75-123.

_- "An Argument against the Social Fact Thesis", en Law and Philosophy, no 27, 2008, pp. 445-504.

Von Wright, Georg H., "Truth-logics", en Logique et Analyse, no 30, 1987, pp. 311-334. 